\title{
Immunokinetics in severe pneumonia due to influenza virus and bacteria coinfection in mice
}

\author{
M. Seki*, K. Yanagihara*, Y. Higashiyama*, Y. Fukuda*, Y. Kaneko*, H. Ohno*, Y. Miyazaki*, \\ Y. Hirakata*, K. Tomono*, J. Kadota*, T. Tashiro*, S. Kohno*\#
}

Immunokinetics in severe pneumonia due to influenza virus and bacteria coinfection in mice. M. Seki, K. Yanagihara, Y. Higashiyama, Y. Fukuda, Y. Kaneko, H. Ohno, Y. Miyazaki, Y. Hirakata, K. Tomono, J. Kadota, T. Tashiro, S. Kohno. (C) ERS Journals Ltd 2004.

ABSTRACT: Coinfections of bacteria and influenza are a major cause of excessive mortality during influenza epidemics. However, the mechanism of the synergy between influenza virus and bacteria are poorly understood.

In this study, mice were inoculated with influenza virus, followed 2 days later by inoculation with Streptococcus pneumoniae. The kinetics of viral titres, bacterial numbers and the immune response (cytokine and chemokine production) were also analysed.

Short-term survival correlated with pathological changes in the lungs of infected mice. Influenza virus or $S$. pneumoniae infection alone induced moderate pneumonia; however, severe bronchopneumonia with massive haemorrhage in coinfected mice, which caused death of these mice $\sim 2$ days after inoculation with $S$. pneumoniae, was noted. Intrapulmonary levels of inflammatory cytokines/chemokines, type-1 T-helper cell cytokines and Toll-like receptors, and the related mitogen-activated protein kinase signalling molecules (phosphorylated extracellular signal-regulated kinase $\mathbf{- 1}$ and $\mathbf{- 2}$, p38 and c-Jun N-terminal kinase), were increased in coinfected mice.

These results suggest that immune mediators, including cytokines and chemokines, through Toll-like receptors/mitogen-activated protein kinase pathways, play important roles in the pathology of coinfection caused by influenza virus and Streptococcus pneumoniae.

Eur Respir J 2004; 24: 143-149.
*Second Dept of Internal Medicine, Nagasaki University School of Medicine, and ${ }^{\#}$ Dept of Molecular Microbiology and Immunology, Nagasaki University Graduate School of Biomedical Science, Nagasaki, Japan.

Correspondence: K. Yanagihara

Second Dept of Internal Medicine

Nagasaki University School of Medicine

1-7-1 Sakamoto

Nagasaki-shi

852-8501 Japan

Fax: 81958497285

E-mail: kyana-ngs@umin.ac.jp

Keywords: Cyclooxygenase-2

cytokine

influenza virus

mitogen-activated protein kinase

Streptococcus pneumoniae

Toll-like receptors

Received: November 112003

Accepted after revision: February 172004
Influenza virus infection is one of the most pandemic infectious diseases in the world [1]. In the USA, $>20,000$ influenza-associated deaths were reported in each of the nine epidemics between 1972 and 1992, and four of these epidemics each resulted in $>40,000$ influenza-associated deaths. In general, influenza virus infection can induce bronchitis and pneumonia, but severe lethal pneumonia is usually seen when complications involve bacterial infections. Hence, coinfections of bacteria and influenza are considered one of the major factors of morbidity and mortality during influenza virus epidemics [2-4].

The great "Spanish flu" influenza pandemic of 1918/1919 killed $\geqslant 20$ million people, making it the worst infectious pandemic in history. Recent studies regarding this pandemic have revealed that most deaths occurred among young adults, a group in which the influenza-related mortality rate is usually very low, and pathology sections from these cases often exhibited massive alveolar haemorrhage and pulmonary oedema [5-7]. In these cases, the majority of sections showed acute lobular pneumonia with massive neutrophil infiltration, suggesting death by acute bacterial pneumonia, one of the most common sequelae of the pandemic. These findings suggest that the combination of influenza virus infection and bacterial infection may play a critical role in inducing severe lung damage and pneumonia in pandemic influenza virus infection.

LEVINE et al. [8] recently investigated the mechanisms of defective pulmonary clearance of Streptococcus pneumoniae using influenza virus-infected mice. They infected mice with S. pneumoniae 7 days after influenza virus inoculation, and demonstrated that prior exposure to influenza virus altered neutrophil function and the lung inflammatory environment, leading to defective $S$. pneumoniae clearance. In an in vitro study, ENGELICH et al. [9] showed that combined infection caused a marked reduction in the survival of neutrophils, which was due to increases in annexin $\mathrm{V}$ binding and caspase3 activation, and also showed that combination infection with influenza virus and $S$. pneumoniae caused significantly more hydrogen peroxide production than either infection alone. Moreover, a detailed study in a viral/bacterial synergistic mouse model was reported, which implicated a receptormediated pathway and other mechanisms in the lethal synergism between influenza virus and bacteria [10].

In the present study, mice were inoculated with influenza virus, followed 2 days later, the day of peak viral titre [11], by inoculation with $S$. pneumoniae, and short-term survival and fulminant pathological changes in the lungs observed. The kinetics of viral titres, bacterial numbers and immune responses, such as cytokines and chemokines, in the lungs of these mice were analysed. Some critical immune mediators, including Toll-like receptor (TLR)/mitogen-activated protein kinase (MAPK) signalling, cyclooxygenase (COX) expression and prostaglandin $\mathrm{E}_{2}\left(\mathrm{PGE}_{2}\right)$ production were then focused on and analysed. 


\section{Materials and methods}

\section{Viral preparation}

A mouse-adapted A-strain influenza virus (strain A/PR8/34; haemagglutinin type 1 , neuraminidase type 1 (H1N1)) was used in the present experiments. Madin-Darby canine kidney cells were used for maintenance of influenza virus and plaque formation assays to analyse viral titres. Influenza virus were cultured in these cells for a few days and the supernatants collected and stored at $-80^{\circ} \mathrm{C}$ as viral stocks until used in experiments.

\section{Bacteria preparation}

Penicillin-resistant S. pneumoniae-187 serotype 19 (isolated at Nagasaki University, Nagasaki, Japan) was used. Bacteria were incubated in brain/heart infusion broth overnight at $37^{\circ} \mathrm{C}$, and harvested by centrifugation for $10 \mathrm{~min}$ at $10,000 \times g$. The harvested bacteria were suspended in phosphate-buffered saline (PBS; $\mathrm{pH} 7.2-7.4)$ at a concentration of $1 \times 10^{8}$ colony-forming units $(\mathrm{cfu}) \cdot \mathrm{mL}^{-1}$, as determined by colony-forming assay on blood agar plates.

\section{Inoculation of mice and sampling}

Male 6-week-old CBA/J specific pathogen-free mice, weighing $30-35 \mathrm{~g}$, were purchased from Japan Charles River, Inc. (Tokyo, Japan). For survival study, 40 animals were divided into four treatment groups: influenza virus- and $S$. pneumoniae-infected mice, influenza virus-infected mice, $S$. pneumoniae-infected mice, and controls. Each group comprised 10 mice. Mice in the influenza virus-infected group were anaesthetised with $50 \mu \mathrm{g} \cdot \mathrm{kg}$ body weight ${ }^{-1}$ pentobarbital and then inoculated intranasally with $50 \mu \mathrm{L}$ $1 \times 10^{4}$ plaque-forming units $\cdot \mathrm{mL}^{-1}$ influenza virus. In the influenza virus- and $S$. pneumoniae-infected group of mice, $50 \mu \mathrm{L} 1 \times 10^{8} \mathrm{cfu} \cdot \mathrm{mL}^{-1} S$. pneumoniae were also inoculated intranasally 2 days after inoculation of influenza virus. The inoculum size of $S$. pneumonia was based on that used in a previous study [12]. Singly infected mice and controls consisted of mice inoculated singly with a dose of either organism and an equal volume of diluent (Roswell Park Memorial Institute (RPMI) 1640 medium), respectively. The mice were observed for 2 weeks for survival studies. The lungs and heart blood were collected as biological samples at $0,3,6$, 12,24 and $48 \mathrm{~h}$ after $S$. pneumoniae infection $(\mathrm{n}=4$ in each group). Bronchoalveolar lavage (BAL) and staining of cells in BAL fluid (BALF) were performed as described previously [13]. Paraffin embedding and tissue staining with haematoxylin and eosin were performed using standard methodologies.

\section{Analysis of Toll-like receptor and cyclooxygenase-2 expression}

Total ribonucleic acid (RNA) was extracted from lung homogenates or cells using ISOGEN (Nippongene, Tokyo, Japan). In order to prepare lung homogenates, lungs were homogenised in $1 \mathrm{~mL}$ PBS using a glass tissue homogeniser (Takashima, Tokyo, Japan) for $60 \mathrm{~s}$ in an ice-cold water bath. Reverse transcriptase polymerase chain reaction (PCR) was performed using the First Strand \& Detection kit (Life Technology, Gaithersburg, MD, USA). Mice TLR and hypoxanthine phosphoribosyltransferase (HPRT) complementary deoxyribonucleic acids were amplified using the specific primers listed in table 1. The specific primers used for amplifications of Cox-1 and -2 were as previously reported by GRAF et al. [14]. Expression levels of mouse HPRT were used as an internal control. PCR was performed for 38 cycles for TLR 2 and TLR 4, 35 cycles for TLR 5 and TLR9, 30 cycles for TLR 3 , and 25 cycles for HPRT. One PCR cycle consisted of $1 \mathrm{~min}$ at $94^{\circ} \mathrm{C}, 1 \mathrm{~min}$ at $55^{\circ} \mathrm{C}$ and $1 \mathrm{~min}$ at $72^{\circ} \mathrm{C}$. The signal intensity of each band on Western blotting was analysed by AlphaImager (Alpha-Innotech Co., San Leandro, CA, USA).

\section{Western blot analysis}

The proteins in the lung homogenates were separated by sodium dodecylsulphate-polyacrylamide gel electrophoresis and transferred on to polyvinylidene difluoride membranes (Millipore, Bedford, MA, USA). The membranes were blocked with $5 \%$ skimmed milk in tris(hydroxymethyl)aminomethane (Tris)-buffered saline ( $\mathrm{pH}$ 7.2-7.4) containing $0.05 \%$ Tween-20 (TBS-T) and incubated with rabbit antibodies directed against either intact extracellular signalregulated kinase-1 and -2 (ERK-1/-2), p38 or c-Jun N-terminal kinase (JNK) or the phosphorylated (activated) forms of these kinases (Cell Signalling Technology, Beverly, MA, USA; each diluted 1:1,000) for $1 \mathrm{~h}$ at room temperature. The membranes were then washed with TBS-T and incubated with horseradish peroxidase-conjugated antirabbit immunoglobulin G (Santa Cruz Technology, Santa Cruz, CA, USA; diluted 1:5,000) for $1 \mathrm{~h}$ at room temperature. After two additional washes, the signals were developed with ECL-plus (Amersham, Arlington Heights, IL, USA).

\section{Enzyme-linked immunosorbent assay}

Concentrations of tumour necrosis factor- $\alpha$ (TNF- $\alpha$ ), interleukin (IL)-4, $-5,-6$ and -12 , interferon gamma (IFN- $\gamma$ ), macrophage inflammatory protein (MIP) 2, regulated on activation, normal T-cell expressed and secreted (RANTES), and $\mathrm{PGE}_{2}$ in aqueous lung extracts were assayed by enzyme-linked immunosorbent assay using mouse Quantikine Kits (R\&D Systems, Inc., Minneapolis, MN, USA). In order to prepare aqueous lung extracts, the homogenates were centrifuged for $60 \mathrm{~min}$ at $2,000 \times g$ at $4^{\circ} \mathrm{C}$ [15]. Supernatants were collected and used in the assay.

Table 1. - Primers used for amplification of mouse Toll-like receptor (TLR) and hypoxanthine phosphoribosyltransferase (HPRT) complementary deoxyribonucleic acids

\begin{tabular}{ccc}
\hline & Primer pair & Size bp \\
\hline TLR2 & 5'-TGGAGAAGGTGAAGCCATTC-3' & 424 \\
& 5'-AAGAACAAAGAAAATGAGTC-3' & \\
TLR3 & 5'-ACATCTCAAAAATAGCAAAT-3' & 267 \\
& 5'-TACGAAGAGGGCGGAAAGGT-3' & \\
TLR4 & 5'-GAATACCTCCTTAGTGTTGG-3' & 648 \\
& 5'-TCTTCCTCTGCTGTTGCTC-3' & \\
TLR5 & 5'-CCAGCTCCAGTTTCTCATCT-3' & 554 \\
& 5'-CCTCTTCATCGCAGTCTTCG-3' & \\
TLR9 & 5'-GTCAGACAATCGCATCAGTG-3' & 257 \\
& 5'-AGTTGTGGCTCAGGCTAAGA-3' & \\
HPRT & 5'-AAGCAGTACAGCCCCAAAAT-3' & 392 \\
& 5'-CATAGTGCAAATCAAAAGTC-3' & \\
\hline
\end{tabular}

bp: base pair; T: thymine; G: guanine; A: adenine; C: cytosine. 


\section{Image and statistical analyses}

The signal intensity of each band on Western blot analysis was determined using an AlphaImager. Data are presented as mean \pm SEM. Differences between groups were examined for significance using an unpaired t-test. Kaplan-Meier analysis was used for analysis of survival rates. Differences in bacterial numbers and viral titres were tested by geometric analysis. A p-value of $<0.05$ denoted a significant difference.

\section{Results}

\section{Short-term survival rates}

In order to assess the infection outcome, short-term survival was analysed in mice that were coinfected with influenza virus and $S$. pneumoniae (fig. 1). In the controls (mock-infected mice), no deaths were observed during the 14day observation period. In contrast, $30 \%$ of mice infected with both influenza virus and $S$. pneumoniae died on the first day and $60 \%$ were dead by day 2 . All coinfected mice died within 3 days of $S$. pneumoniae inoculation. Survival of coinfected mice was significantly shorter than that of mock- or singly infected mice $(\mathrm{p}<0.01)$.

\section{Severe pneumonia in coinfected mice}

Pathological examination of the lungs of coinfected mice showed severe bronchopneumonia with massive haemorrhage (fig. 2d), whereas the lungs of mice infected with a single type of pathogen exhibited only moderate pneumonia (fig. $2 \mathrm{~b}$ and c) on day 2. The total number of cells in BALF was also significantly higher in coinfected mice compared to singly infected mice (table 2). Coinfected lungs were filled with inflammatory cells and blood, and almost the entire airspace was obliterated (fig. 2h, 1 and p). The BALF data suggested neutrophil accumulation and showed the highest gain among inflammatory cells in the lungs of coinfected mice (table 2). In contrast, pulmonary invasion of inflammatory cells was mild in singly infected mice (influenza: fig. $2 \mathrm{f}, \mathrm{j}$ and $\mathrm{n}$; S. pneumoniae: fig. $2 \mathrm{~g}$, k and o). The lungs of mock-infected mice showed no inflammation (fig. $2 \mathrm{a}, \mathrm{e}, \mathrm{i}$ and $\mathrm{m}$ ).

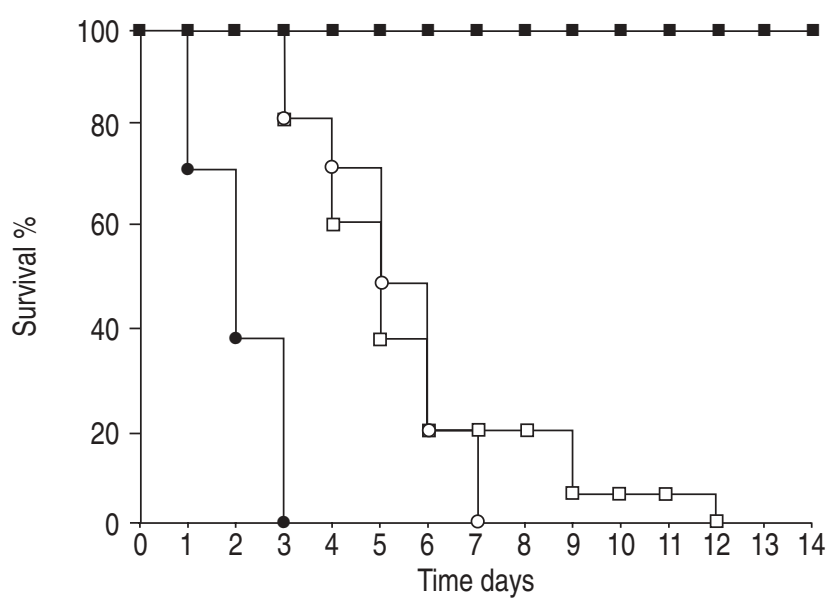

Fig. 1.-Survival rates of mice infected with influenza virus and/or Streptococcus pneumoniae. Mice were infected with influenza virus 2 days before $S$. pneumoniae instillation, which occurred on day 0. Each group comprised 10 mice $(\bullet$ : doubly infected mice; $\bigcirc$ : influenza virus-infected mice; $\square$ : S. pneumoniae-infected mice; $\mathbf{\square}$ : mock-infected mice). The survival rate was significantly lower in mice infected with both influenza virus and $S$. pneumoniae than in singly or mock-infected mice $(\mathrm{p}<0.01)$.
The number of $S$. pneumoniae in the lungs showed a $10-100$-fold increase in coinfected mice at $12(\mathrm{p}<0.05), 24$ and $48(\mathrm{p}<0.01) \mathrm{h}$, compared with singly infected mice. However, viral titres in coinfected lungs were almost identical to those of mice infected with influenza virus alone (data not shown).

\section{Effect of infection type on inflammatory cytokines in the lungs}

The concentrations of these inflammatory cytokines were compared between coinfected mice and singly or mockinfected mice on day 2 (table 3 ). The concentrations of all inflammatory cytokines/chemokines, including TNF- $\alpha$, IL-6, MIP-2 and RANTES, were significantly higher in the lungs of coinfected mice than in the lungs of either singly or mock-infected mice (table 3).

\section{Type-1/type-2 T-helper cell balance in coinfected lungs}

In the present coinfected mice, levels of IFN- $\gamma$, type- 1 T-helper cell (Th1) cytokines and IL-12, which directs the induction of Th1, were significantly higher than in singly and mock-infected mice (table 3 ). In contrast, type-2 T-helper cell (Th2) cytokines, such as IL-4 and -5, were not induced or suppressed, respectively, in the lungs of coinfected mice, compared to singly and mock-infected mice (table 3 ).

\section{Expression of Toll-like receptor messenger ribonucleic acids in coinfected lungs}

The expression levels of TLR messenger RNA (mRNA) (TLR2, -3, -4, -5 and -9) in the lungs of coinfected mice were examined on day 2 (fig. 3; table 4). Both influenza and $S$. pneumoniae infections induced upregulation of TLR2 and -4 mRNA. Greater induction of TLR2 and -4 mRNA were also observed in the lungs of coinfected mice, compared to either influenza- or S. pneumoniae-infected mice. Upregulation of TLR5 and -9 mRNA in the lungs of coinfected mice was weak, and almost identical in influenza- and S. pneumoniaeinfected mice. The expression level of TLR 3 mRNA did not change with either coinfection or single infection.

\section{Mitogen-activated protein kinase signalling in coinfected lungs}

Activation of ERK-1/-2 and p38, and to a lesser extent of JNK, was observed in the lungs of $S$. pneumoniae- and influenza virus-infected mice, compared to mock-infected mice. Very strong activation of these MAPK molecules was noted in coinfected lungs (fig. 4; table 5).

\section{Cyclooxygenase-2 production in the lungs and its effects on coinfected mice}

COX-2 mRNA was upregulated in the lungs of both influenza virus- and $S$. pneumoniae-infected mice, compared to mock-infected mice (fig. 5a; table 4). Significant expression of COX-2 mRNA was observed in the lungs of coinfected mice, compared to those of singly and mock-infected mice. In contrast to COX-2 mRNA, COX-1 mRNA levels did not change and there was constitutive expression in all samples (fig. 5a; table 4). The effect of COX-2 on $\mathrm{PGE}_{2}$ production was also examined. There was a significant increase in $\mathrm{PGE}_{2}$ production in the lungs of coinfected mice compared to singly and mock-infected mice (fig. 5b). 

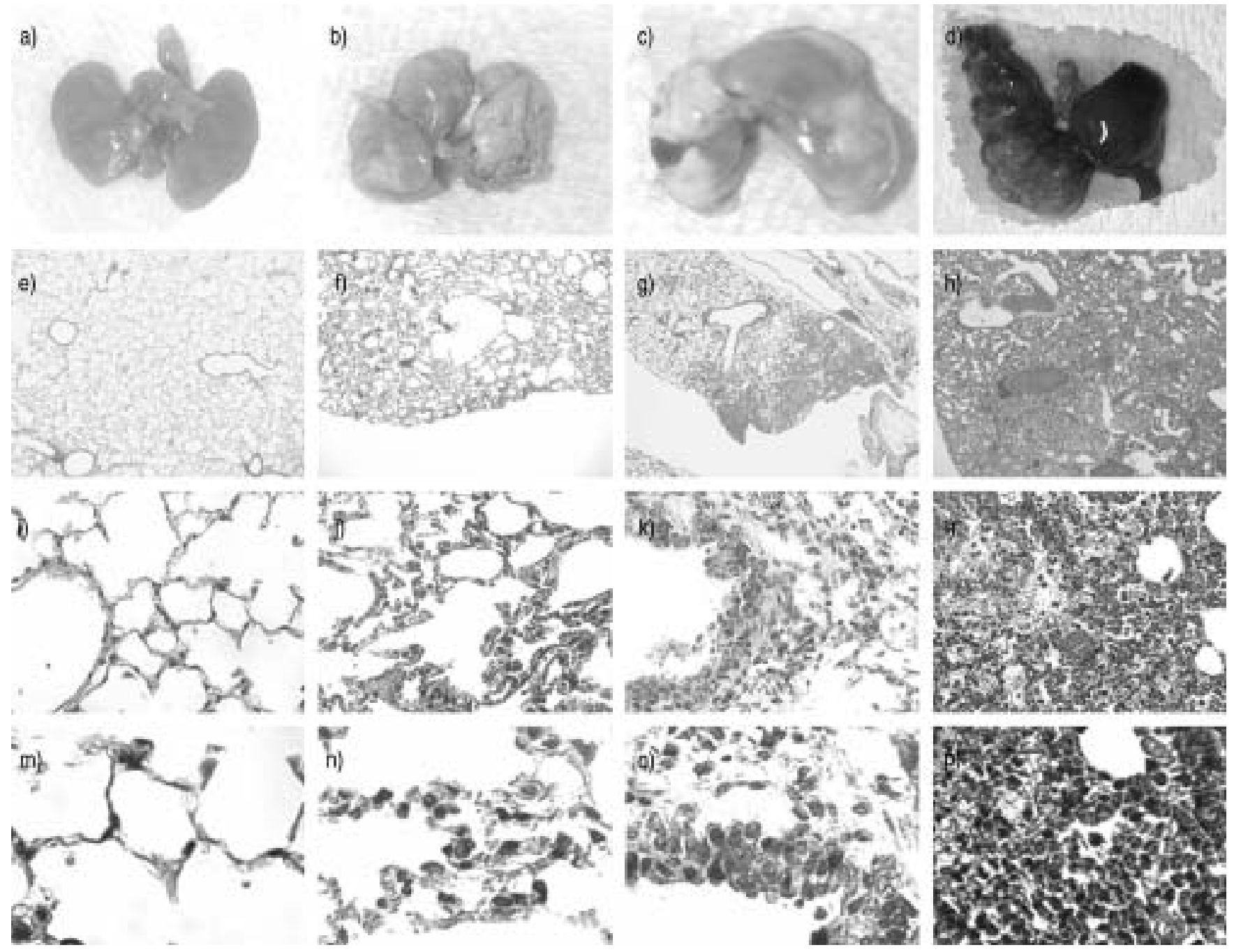

Fig. 2. - Pathological analysis of the lungs of mice infected with influenza virus and/or Streptococcus pneumoniae. Lungs were collected 2 days after S. pneumoniae inoculation. a-d) photographs of whole lungs; and haematoxylin and eosin-stained tissue sections at magnifications of e-h) $\times 40, \mathrm{i}-1) \times 400$ and $\mathrm{m}-\mathrm{p}) \times 1,000$. a, e, i, m) Mock-infected (control) mice, b, f, j, n) influenza virus-infected mice, c, g, k, o) S. pneumoniaeinfected mice and $\mathrm{d}, \mathrm{h}, \mathrm{l}, \mathrm{p}$ ) doubly infected mice.

Table 2. - Inflammatory cells in the bronchoalveolar lavage fluid of mice infected with influenza virus and/or Streptococcus pneumoniae on day 2

Mock

Influenza

Streptococcus pneumoniae

Coinfection

Cell density

$10^{4}$ cells $\cdot \mathrm{mL}^{-1}$

Total cells

Neutrophils

Macrophages

Lymphocytes

$\begin{array}{cc}5.0 \pm 0.4 & 60.2 \pm 4.6^{*} \\ 0 & 20.1 \pm 1.1^{*} \\ 5.0 \pm 0.4 & 35.0 \pm 2.2^{*} \\ 0 & 4.1 \pm 0.3^{*}\end{array}$
$110.8 \pm 10.2^{*, \#}$
$72.5 \pm 6.3^{*, \#}$
$38.3 \pm 3.9^{*}$

0

Data are presented as mean \pm SEM $(\mathrm{n}=4) .{ }^{*}: \mathrm{p}<0.05$ versus mock-infected mice; ${ }^{*}: \mathrm{p}<0.05$ versus influenza virus-infected mice; ${ }^{\top}$ : $\mathrm{p}<0.05$ versus $S$. pneumoniae-infected mice.

\section{Discussion}

In the present study, a mouse model of coinfection with influenza virus and $S$. pneumoniae was used, and significant short-term survival of coinfected mice found, due to severe bronchopneumonia and massive lung haemorrhage. These lethal pathological changes progressed rapidly $\sim 48 \mathrm{~h}$ after $S$. pneumoniae inoculation. On microbiological analysis, counts of $S$. pneumoniae in the lungs of mice with a preceding influenza virus infection were 10-100-fold higher than in the lungs of mice infected with $S$. pneumoniae alone. However, the titres of influenza virus were similar in coinfected mice and in mice infected with influenza virus alone. These data suggest that the pathogenesis of severe lung damage is probably not dependent on simple synergy between the agents. The host immune response is one of the most important factors in determining lung pathology in coinfected mice, and infection by influenza virus may favour the growth of bacteria, 
Table 3.-Inflammatory cytokine and chemokine concentrations in the lungs of mice infected with influenza virus and/or Streptococcus pneumoniae

\begin{tabular}{llccc}
\hline & Mock & Influenza & Streptococcus pneumoniae & Coinfection \\
\hline TNF- $\alpha \mathrm{ng} \cdot \mathrm{mL}^{-1}$ & $53.8 \pm 15.8$ & $161.1 \pm 42.0$ & $164.7 \pm 35.1$ & $308.4 \pm 60.6^{* *}$ \\
IL-6 ng $\cdot \mathrm{mL}^{-1}$ & $54.2 \pm 1.1$ & $65.1 \pm 7.6$ & $59.4 \pm 5.3$ & $267.5 \pm 40.4^{* *}$ \\
MIP-2 $\mathrm{ng} \cdot \mathrm{mL}^{-1}$ & $45.9 \pm 2.9$ & $184.7 \pm 7.6$ & $241.5 \pm 17.5$ & $594.7 \pm 43.7^{* * * * *}$ \\
RANTES ng $\cdot \mathrm{mL}^{-1}$ & $99.3 \pm 7.0$ & $107.4 \pm 30.0$ & $162.8 \pm 14.4$ & $385.7 \pm 65.5^{* *}$ \\
Th1 & $31.1 \pm 2.1$ & $36.3 \pm 2.1$ & $165.4 \pm 14.5$ & $436.2 \pm 15.9^{* *}$ \\
IFN- $\gamma \mathrm{ng} \cdot \mathrm{mL}^{-1}$ & $99.1 \pm 21.4$ & $146.0 \pm 10.4$ & $328.9 \pm 41.7$ & $476.3 \pm 36.5^{* * * * *}$ \\
IL-12 $\mathrm{ng} \cdot \mathrm{mL}^{-1}$ & $19.1 \pm 1.4$ & $20.1 \pm 4.4$ & $18.1 \pm 3.4$ & $30.5 \pm 2.3$ \\
Th2 & $56.8 \pm 1.4$ & $56.7 \pm 4.4$ & $60.0 \pm 3.9$ & $57.5 \pm 5.1$ \\
IL-4 $\mathrm{ng} \cdot \mathrm{mL}^{-1}$ & $\mathrm{IL}-5 \mathrm{ng} \cdot \mathrm{mL}^{-1}$ & & & \\
\hline
\end{tabular}

Data are presented as mean \pm SEM $(n=4)$. TNF- $\alpha$ : tumour necrosis factor- $\alpha$; IL: interleukin; MIP: macrophage inflammatory protein; RANTES: regulated on activation, normal T-cell expressed and secreted; Th: T-helper cell; IFN- $\gamma$ : interferon gamma. *: p $<0.05$ versus S. pneumoniae-infected mice; **: $\mathrm{p}<0.01$ versus singly or mock-infected mice; ***: $\mathrm{p}<0.001$ versus influenza virus- and mock-infected mice.
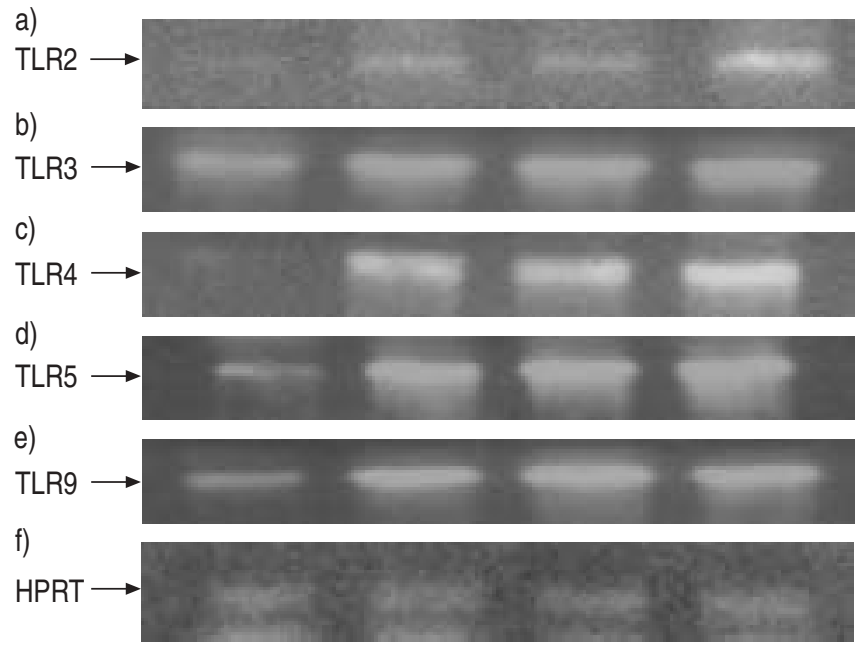

$\begin{array}{llll}1 & 2 & 3 & 4\end{array}$

Fig. 3. - Toll-like receptor (TLR) messenger ribonucleic acid expression in the lungs of mice infected with influenza virus and/or Streptococcus pneumoniae on day 2, analysed by reverse transcriptase polymerase chain reaction with hypoxanthine phosphoribosyltransferase (HPRT) as internal control: a) TLR2, b) TLR3, c) TLR4, d) TLR5, e) TLR9 and f) HPRT (lane 1: mock-infected; lane 2: influenza virus-infected; lane 3: S. pneumoniae-infected; lane 4: doubly infected; relative densities are shown in table 4). The experiment was repeated three times; representative results are shown.

Table 4.-Expression ${ }^{\#}$ of Toll-like receptor (TLR) and cyclooxygenase (COX)-1 and -2 messenger ribonucleic acid in the lungs of mice infected with influenza virus and/or Streptococcus pneumoniae

\begin{tabular}{lcccc}
\hline & Mock & Influenza & $\begin{array}{c}\text { Streptococcus } \\
\text { pneumoniae }\end{array}$ & Coinfection \\
\hline TLR2 & $0.4 \pm 0.1$ & $1.0 \pm 0.2$ & $0.9 \pm 0.2$ & $1.9 \pm 0.4$ \\
TLR3 & $1.1 \pm 0.2$ & $1.2 \pm 0.2$ & $1.2 \pm 0.3$ & $1.0 \pm 0.2$ \\
TLR4 & $0.2 \pm 0.1$ & $2.0 \pm 0.3$ & $1.8 \pm 0.3$ & $2.4 \pm 0.3$ \\
TLR5 & $0.8 \pm 0.2$ & $1.8 \pm 0.3$ & $1.9 \pm 0.2$ & $2.0 \pm 0.4$ \\
TLR9 & $1.0 \pm 0.3$ & $1.9 \pm 0.4$ & $1.9 \pm 0.3$ & $2.2 \pm 0.3$ \\
COX-1 & $1.0 \pm 0.2$ & $0.6 \pm 0.3$ & $0.8 \pm 0.2$ & $1.0 \pm 0.2$ \\
COX-2 & $0.2 \pm 0.1$ & $0.4 \pm 0.3$ & $1.8 \pm 0.3$ & $8.6 \pm 2.6$ \\
\hline
\end{tabular}

Data are presented as mean \pm SEM $(n=4) .{ }^{*}$ : relative to hypoxanthine phosphoribosyltransferase band density on day 2 .

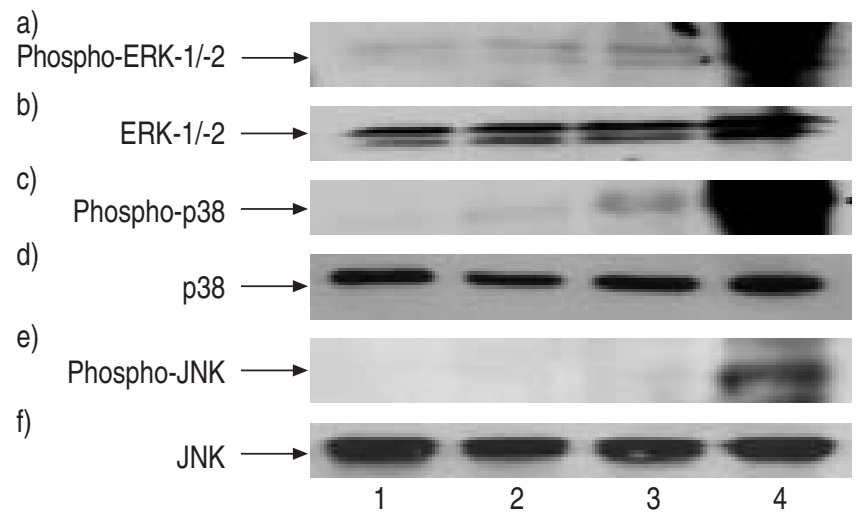

Fig. 4. - Activation of $a, b)$ extracellular signal-regulated kinase-1 and -2 (ERK-1/-2), c, d) p38 and e, f) c-Jun N-terminal kinase (JNK), signalling factors in the Toll-like receptor-mediated cytokine production pathway, in the lungs of mice infected with influenza virus and/ or Streptococcus pneumoniae on day 2. Levels of activation were analysed by Western blotting, using antibodies directed against each: $\mathrm{a}, \mathrm{c}, \mathrm{e})$ phosphorylated (activated) form and $\mathrm{b}, \mathrm{d}, \mathrm{f}$ ) intact form (lane 1: mock-infected; lane 2: influenza virus-infected; lane 3: S. pneumoniae-infected; lane 4: doubly infected; the ratios of phosphorylated to nonphosphorylated protein are shown in table 5). The experiment was repeated three times; representative results are shown.

resulting in a secondary bacterial infection and subsequent severe lung damage. Recent in vitro studies have demonstrated that influenza A virus-infected HEp-2 cells exhibit significantly greater binding to $S$. pneumoniae strains than uninfected cells [16]. It has been proposed that influenza virus infection may activate the epithelium and upregulate cytokine production and receptors for platelet activating factor. These influenza virus-induced mechanisms may underlie the enhanced bacterial adherence and increased tissue damage in the lungs. Therefore, the present study focused on host immune responses and analysis of inflammatory molecules in the mice used.

The present results revealed significant increases in intrapulmonary pro-inflammatory and inflammatory cytokine/chemokine concentrations. Among these cytokines and chemokines, the concentrations of TNF- $\alpha$, IL-6, MIP-2 (functionally similar to IL-8 in human) and RANTES increased significantly in the lungs of coinfected mice, compared to either mock- or singly infected mice. These results suggest that TNF- $\alpha$, IL-6, MIP-2 and RANTES play important roles in the pathological changes in the lungs of mice with severe pneumonia. 
Table 5. - Activation" of extracellular signal-regulated kinase1 and -2 (ERK-1/-2), p38 and c-Jun N-terminal kinase (JNK) in the lungs of mice infected with influenza virus and/or Streptococcus pneumoniae

\begin{tabular}{lcccc}
\hline & Mock & Influenza & $\begin{array}{c}\text { Streptococcus } \\
\text { pneumoniae }\end{array}$ & Coinfection \\
\hline ERK-1/-2 & $0.2 \pm 0.1$ & $0.4 \pm 0.2$ & $0.8 \pm 0.2$ & $3.2 \pm 0.4$ \\
p38 & $0.1 \pm 0.1$ & $0.2 \pm 0.2$ & $0.8 \pm 0.3$ & $4.4 \pm 0.6$ \\
JNK & $0.1 \pm 0.1$ & $0.1 \pm 0.2$ & $0.2 \pm 0.1$ & $1.6 \pm 0.4$ \\
\hline
\end{tabular}

Data are presented as mean \pm SEM $(n=4)$. ${ }^{*}$ : ratio of phosphorylated to nonphosphorylated protein by Western blot band density on day 2 .
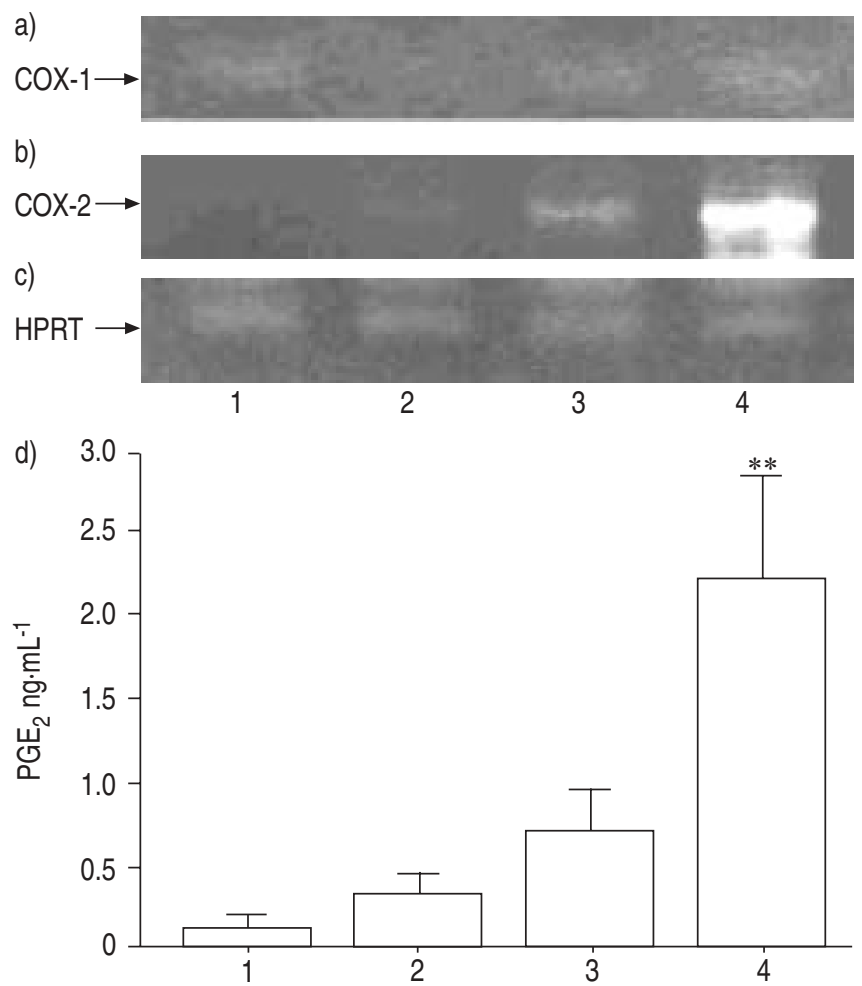

Fig. 5. - Expression of a) cyclooxygenase (COX)-1, b) COX-2 and c) hypoxanthine phosphoribosyltransferase (HPRT) messenger ribonucleic acid (mRNA), analysed by reverse transcriptase polymerase chain reaction with HPRT as internal control (relative densities are shown in table 4), and d) prostaglandin $\mathrm{E}_{2}\left(\mathrm{PGE}_{2}\right)$ in the lungs of mice infected with influenza virus and/or Streptococcus pneumoniae on day 2 (1: mock-infected; 2: influenza virus-infected; 3: S. pneumoniaeinfected; 4: doubly infected). The mRNA expression experiment was repeated three times; representative results are shown. $\mathrm{PGE}_{2}$ data are presented as mean \pm SEM. ${ }^{* *}: \mathrm{p}<0.01$ versus all other groups.

Moreover, the present results revealed increases in Th1 cytokine levels, but no significant changes in Th2 cytokine levels, in the lungs of coinfected mice. In general, Th1 cytokines are known to exert protective and antiviral effects. However, Th2 cytokines exhibit inhibitory effects on viral clearance. The balance between Th1 and Th2 cytokines seems to be involved in the pathogenesis of severe lung damage observed in the present study.

In order to evaluate the relationships between cytokines and the pathological changes, the related molecules TLR and MAPK were investigated in terms of mRNA expression and activation, respectively, in coinfected lungs. The production of cytokines and chemokines in response to components of microbiological agents is stimulated by TLRs and mediated by MAPK signalling [17, 18]. Of TLR2, $-3,-4,-5$ and -9 , TLR2 mRNA showed the most specific induction in coinfected lungs. TLR2 is known as the recognition receptor for peptidoglycans of Gram-positive bacteria, including $S$. pneumoniae [19]. Furthermore, strong activation of MAPK-related molecules, such as ERK-1/-2, p38 and JNK, was observed. These data suggest that TLR, especially TLR2 stimulation, and MAPK signalling cytokine secretion pathways may be involved in the severe lung damage noted in coinfected mice.

The potential role of other inflammatory mediators, COXs, which convert arachidonic acid to prostaglandins, was also analysed [20]. Of the two known COX isoforms, COX-1 is constitutively expressed in most cells and is thought to be important for normal physiological functions. Conversely, COX-2 is usually expressed at low basal levels and is rapidly induced by inflammatory stimulation. It was reported that COX-2 expression is dependent on MAPK signalling and IL$1 \beta$ secretion $[21,22]$. In the present study, COX-2 mRNA expression was significantly increased in coinfected lungs, consistent with the pathological changes. COX-2 expression has been detected in fatal pneumococcal pneumonia and allergic inflammation in guinea-pig lungs. In the present study, significant upregulation of COX-2 mRNA was found in samples from coinfected mice, compared to those from singly or mock-infected mice. Significantly high concentrations of $\mathrm{PGE}_{2}$ were also noted in coinfected lungs, compared to either mock- and/or singly infected lungs, suggesting that COX-2 and COX-2-stimulated $\mathrm{PGE}_{2}$ production might be important mediators of the severe pneumonia in coinfected mice.

In conclusion, intranasal inoculation of influenza virus and Streptococcus pneumoniae in mice resulted in severe lethal pneumonia with lung haemorrhage. These pathological changes were initiated by the infectious agents and regulated by host immune responses, including the Toll-like receptormediated signalling pathway. Administration of cyclooxygenase-2 inhibitors may protect against severe inflammation. Further studies are necessary to elucidate the molecular mechanisms of fulminant lung damage in influenza virus and Streptococcus pneumoniae coinfection.

Acknowledgements. The authors are grateful to M. Harada for excellent technical support, and to F.G. Issa (Word-Medex Pty Ltd, Sydney, Australia) for careful reading and editing of the manuscript.

\section{References}

1. American Thoracic Society. Guidelines for the management of adults with community-acquired pneumonia. Am J Respir Crit Care Med 2001; 63: 1730-1754.

2. Glezen WP. Serious morbidity and mortality associated with influenza virus epidemics. Epidemiol Rev 1982; 4: 25-44.

3. Schwarzmann SW, Adler JL, Sullivan RL, Marine WM. Bacterial pneumonia during the Hong Kong influenza epidemic of 1968-1969. Arch Intern Med 1971; 127: 1037-1041.

4. Seemungal TR, Harper-Owen $\mathrm{R}$, Bhowmik $\mathrm{A}$, et al. Respiratory viruses, symptoms, and inflammatory markers in acute exacerbations and stable chronic obstructive pulmonary disease. Am J Respir Crit Care Med 2001; 164 : $1618-1623$.

5. Reid AH, Fanning TG, Hultin JV, Taubenberger JK. Origin and evolution of the 1918 "Spanish" influenza virus hemagglutinin gene. Proc Natl Acad Sci USA 1999; 96: 1651-1656. 
6. Taubenberger JK. Influenza virus hemagglutinin cleavage into HA1, HA2: no laughing matter. Proc Natl Acad Sci USA 1998; 95: 9713-9715.

7. Taubenberger JK, Reid AH, Krafft AE, Bijwaard KE, Fanning TG. Initial genetic characterization of the 1918 "Spanish" influenza virus. Science 1997; 275: 1793-1796.

8. LeVine AM, Koeningsknecht V, Stark JM. Decreased pulmonary clearance of $S$. pneumoniae following influenza A infection in mice. $J$ Virol Methods 2001; 94: 173-186.

9. Engelich G, White M, Hartshorn KL. Neutrophil survival is markedly reduced by incubation with influenza virus and Streptococcus pneumoniae: role of respiratory burst. J Leukoc Biol 2001; 69: 50-56.

10. McCullers JA, Rehg JE. Lethal synergism between influenza virus and Streptococcus pneumoniae: characterization of a mouse model and role of platelet-activating factor receptor. J Inf Dis 2002; 186: 341-350.

11. Hayden FG, Fritz RS, Lobo MC, Alvord WG, Strober W, Straus SE. Local and systemic cytokine responses during experimental human influenza $\mathrm{S}$ virus infection. Relation to symptom formation and host defense. J Clin Invest 1998; 101: 643-649.

12. Otsu $\mathrm{Y}$, Yanagihara $\mathrm{K}$, Fukuda $\mathrm{Y}$, et al. In vivo efficacy of a new quinolone, DQ-113, against Streptococcus pneumoniae in a mouse model. Antimicrob Agents Chemother 2003; 47: 3699-3703.

13. Yanagihara K, Seki M, Cheng P-W. Lipopolysaccharide induces mucus cell metaplasia in mouse lung. Am J Respir Cell Mol Biol 2001; 24: 66-73.

14. Graf BA, Nazarenko DA, Borrello MA, et al. Biphenotypic B/macrophage cells express COX-1 and up-regulate COX-2 expression and prostaglandin $\mathrm{E}_{2}$ production in response to pro-inflammatory signals. Eur $J$ Immunol 1999; 29: 3793-3803.

15. Yanagihara $\mathrm{K}$, Tomono $\mathrm{K}$, Kuroki $\mathrm{M}$, et al. Intrapulmonary concentrations of inflammatory cytokines in a mouse model of chronic respiratory infection caused by Pseudomonas aeruginosa. Clin Exp Immunol 2000; 122: 67-71.

16. El Ahmer OR, Raza MW, Ogilvie MM, Weir DM, Blackwell CC. Binding of bacteria to HEp-2 cells infected with influenza A virus. FEMS Immunol Med Microbiol 1999; 23: 331-341.

17. Akira S, Takeda K, Kaisho T. Toll-like receptors: critical proteins linking innate and acquired immunity. Nature Immunol 2001; 2: 675-680.

18. Pleschka S, Wolff T, Ehrhardt C, et al. Influenza virus propagation is impaired by inhibition of the Raf/MEK/ERK signalling cascade. Nature Cell Biol 2001; 3: 301-305.

19. Yoshimura A, Lien E, Ingalls RR, Tuomanen E, Dziarski R, Golenbock D. Cutting edge: recognition of Grampositive bacterial cell wall components by the innate immune system occurs via Toll-like receptor 2. J Immunol 1999; 163: 1-5.

20. Smith WL, Garavito RM, DeWitt DL. Prostaglandin endoperoxide $\mathrm{H}$ synthases (cyclooxygenases)-1 and -2 . J Biol Chem 1996; 271: 33157-33160.

21. Bonazzi A, Bolla M, Buccellati C, et al. Effect of endogenous and exogenous prostaglandin $\mathrm{E}_{2}$ on interleukin-1 $\beta$-induced cyclooxygenase-2 expression in human airway smoothmuscle cells. Am J Respir Crit Care Med 2000; 162: 2272-2277.

22. Oguma T, Asano K, Shiomi T, et al. Cyclooxygenase-2 expression during allergic inflammation in guinea-pig lungs. Am J Respir Crit Care Med 2002; 165: 382-386. 